\title{
10. Resümee und Schlussbetrachtungen
}

In meiner Studie habe ich den Blick auf ein Phänomen der neuen Zuwanderung der Schweiz gelegt und mich mit dem freiwilligen Engagement von Expatriates im Kanton Zug auseinandergesetzt. Dabei habe ich mich zum einen auf die Expatriates selbst konzentriert und herausgearbeitet, welchen Platz Expatriates im Kanton Zug dem Engagement in ihren alltäglichen Lebenswelten einräumen. Anhand von narrativen Interviews mit Expatriates habe ich vier Expatriate-Typen herausgearbeitet, die sich hinsichtlich ihrer Orientierungen zum freiwilligen Engagement unterscheiden. Bei dieser Typologie spreche ich von einer international-lokalen Selbstverortung, einer internationalen mit punktuell lokaler Selbstverortung, einer Selbstverortung innerhalb der Expatriate-Community sowie einer Suche nach lokaler Selbstverortung. Neben dieser Perspektive der Expatriates habe ich zum anderen die Perspektive etablierter Akteur*innen von zivilgesellschaftlichen Vereinigungen und Organisationen in Zug untersucht, in welchen das Engagement praktiziert und umgesetzt wird. So konnte ich auf Basis von Gruppendiskussionen mit Vereinen rekonstruieren, wie diese Vereine kollektiv mit dem Thema Engagement von Expatriates umgehen. Damit verbunden sind Erkenntnisse darüber, welche Erfahrungen mit dem Engagement von Expatriates in den Vereinen vorliegen und welche kollektiven Umgangsweisen und Erwartungshaltungen sich darin erkennen lassen. Auf Basis von Gruppendiskussionen habe ich für diese kollektive Perspektive der Vereine ebenfalls vier Typen herausgearbeitet, die sich in ihren Orientierungen auf das Engagement von Expatriates unterscheiden; diese habe ich in strukturell-tradierte, ambivalent-adaptive, flexibel-progressive und Expatriate-orientierte Orientierung unterschieden.

Die empirischen Ergebnisse bilden zusammen eine Gesamtschau beider am Engagement beteiligten Seiten und vervollständigen so die qualitativen Forschungen zum zivilgesellschaftlichen Engagement von Expatriates um diese beiden Perspektiven. So bilanziert diese Arbeit aus einer qualitativ ausgerichteten Stossrichtung heraus die wichtigen Erkenntnisse über den Alltag und Engagement von Expatriates, die im Kanton Zug leben. Sie berücksichtigt neben der Perspektive der erforschten Expatriates auch die spezifischen Rahmenbedingungen der Angebotsseite (Freitag et al. 2016: 187), unter denen das Engagement für diese Bevölkerungsgruppe vor Ort überhaupt stattfinden kann.

Ausgehend von den Ergebnissen meiner beiden Teilstudien komme ich im Folgenden zu den Schlussbetrachtungen, die Potenziale für die praktische Verwendung meiner Befunde aufzeigen sollen.

Akzeptanz von unterschiedlichen Lebensweisen von Expatriates mit unterschiedlichem Engagementpotenzial

Ich habe deutlich gemacht, dass es in Zug unterschiedliche Expatriate-Typen gibt, die jeweils unterschiedliche Ansprüche an und Bereitschaften für ein Engagement vor Ort stellen. So konnte ich darlegen, dass es diejenigen gibt, die aus unterschiedlichen Gründen und Motivlagen in der Expatriate-Welt leben und unter ihresgleichen bleiben möchten, das wäre die viel beschriebene Expatriate-Lebenswelt in einer „Bubble“ oder „Parallelwelt“, in der man dann auch

(C) Der/die Autor(en) 2021

M. Störkle, Expatriates und freiwilliges Engagement in der

Schweiz, https://doi.org/10.1007/978-3-658-33043-9_10 
„,unter sich“ in einem meist englischsprachigen Umfeld bleiben und leben kann. Kontakte nach draussen, in die lokale „Schweizer Welt“ können, müssen aber nicht zwangsweise gesucht und gepflegt werden. Weiter gibt es aber noch andere Expatriates, die ihren Radius erweitern, manche mehr, manche weniger. Das sind diejenigen, die nach und nach aktiv werden, ausserhalb der „Bubble“ leben oder zumindest Bereitschaft zeigen, diese ab und zu einmal zu verlassen. Dies sind häufig auch die Expatriates, die schon länger vor Ort leben, bereits etwas Deutsch können oder es lernen wollen. Darüber hinaus verfügen diese im sozialen Nahraum (wie Nachbarschaft oder Quartier) über regelmässige Kontakte zur lokalen Schweizer Wohnbevölkerung und haben meist Kinder vor Ort.

Hinsichtlich der Diskussion um einen (besseren) Einbezug von Expatriates in das lokale Engagement ist es notwendig zu wissen, welches dafür überhaupt die geeignete Zielgruppe ist. So ist es die oben zuletzt angesprochene Gruppe der sogenannten ,long time stayer ${ }^{\text {“50 }}$, die hierbei ins Zentrum des Interesses rücken sollte. Auf ihnen sollte, wenn man über weitere unterstützende Massnahmen der Motivation zum Engagement nachdenkt, der Fokus liegen, um so den Aktiven bzw. den Engagement-Bereiten ein adäquates Umfeld anzubieten. An dieser Stelle möchte ich aber auch betonen, dass es in Zug andere Expatriates gibt, die man kaum erreicht und kaum zu weitergehendem Engagement motivieren kann, insbesondere dann, wenn sie noch jung sind und ohne Familie in die Schweiz kommen. Von diesem Personenkreis ist generell eher wenig Engagementinteresse und -potenzial vor Ort zu erwarten. Das Leben dieser meist klassischen Expatriate-Entsendungen (vgl. z. B. Fechter 2007) dreht sich um die Gestaltung einer internationalen Karriere, die auch einen regelmässigen Wechsel der Wohnorte verlangt. Gerade wenn Expatriates nur für eine kurze Zeitspanne ins Land kommen, kann davon ausgegangen werden, dass neben dem beruflichen Alltag eher wenig Zeit bleibt, sich überhaupt mit Engagement vor Ort zu befassen.

So möchte ich im Rahmen der Diskussionen um Expatriates und Engagement betonen und auch sensibilisieren, dass es ,die Expatriates“ als homogene Gruppe nicht gibt. Es handelt sich dabei vielmehr um eine sehr heterogene Gruppe mit unterschiedlichen internationalen Biografien, Lebensentwürfen und Anspruchshaltungen an das Leben vor Ort. Wenn es nun darum geht, vermehrt Expatriates in das lokal orientierte Engagement miteinzubeziehen, wird es folglich das Ziel sein, die richtigen bzw. diejenigen unter ihnen ausfindig zu machen, die gern aktiv werden und hin und wieder die Expatriate-Welt verlassen wollen.

Expatriate-Clubs in Zug sind wichtige Ausgangspunkte für Aktivität und Engagement für Expatriates in Zug, sowohl im internationalen als auch im lokalen schweizerischen Setting

Im Kanton Zug hat sich eine aktive Expatriate-Infrastruktur entwickelt, in der ankommende und schon länger vor Ort lebende Expatriates vielfältige Angebote vorfinden. Einige dieser sich an Expatriates richtenden Vereine und Initiativen bestehen seit vielen Jahren, im Falle der beiden mit in die Studie einbezogenen Expatriate-Clubs (ZIWC und IMCZ) beinahe 40 Jahre. Wie

\footnotetext{
${ }^{50}$ Allerdings gibt es unter diesen lange vor Ort lebenden Expatriates auch solche, die nach über 30 Jahren noch immer sehr wenig in lokalen schweizerischen Settings angekommen sind, Herr Becker ist so ein Beispiel.
} 
in meiner Arbeit an vielen Stellen gezeigt, sind die darin angebotenen Aktivitäten und Engagementformen sehr gut auf den Lebensalltag vieler Expatriates in Zug angepasst. Somit können diese beiden Clubs als wichtige Player der zivilgesellschaftlichen Engagementlandschaft in Zug betrachtet werden, welche den in Zug lebenden Expatriates die Möglichkeit bieten, an ihrem neuen Wohnort aktiv zu werden bzw. sich zu engagieren.

Wenn es nun darum gehen soll, das Engagement von Expatriates in der Region Zug gesellschaftlich besser nutzbar zu machen, führt letztlich kein Weg an diesen Expatriate-Vereinen vorbei. Vor diesem Hintergrund wäre zu überlegen, wie man die bereits bestehenden Aktivitäten noch systematischer in das öffentliche Leben vor Ort mit einbinden könnte. Die beiden Expatriate-Vereine können dabei als wichtige Schnittstelle vor Ort fungieren, in denen nicht nur die Basis für ein Ankommen und Leben in einer Expatriate-Welt gelegt wird. Es können daraus auch Kontakte und Engagementformen entstehen, die den Aktions- bzw. den Engagementradius erweitern. Dabei wäre es sinnvoll, unter Einbezug der vorhandenen Expatriate-Infrastruktur, den Fachstellen für Integration bei Stadt und Kanton sowie den seit 2012 regelmässig durchgeführten „Let's talk“-Veranstaltungen ${ }^{51}$ weitere regelmässig stattfindende Austauschformen zu konzipieren, an denen sich engagierfreudige Expatriates und Vereine im Raum Zug näherkommen. An solchen Veranstaltungen könnte man also gezielt und systematisch die besonderen Herausforderungen von engagierwilligen und sich bereits engagierenden Expatriates zur Sprache bringen.

Als weitere Schlussfolgerung für eine praxisnahe Verwendung lohnt es sich an dieser Stelle, über einen koordinierten Aufbau von Engagement-Karrieren ausserhalb der Expatriate-Welt nachzudenken. Es wäre zu überlegen, inwieweit die international ausgerichteten Vereine wie der ZIWC und der IMCZ auch selbst systematische Orientierungen für Aktivitäten und Engagement ausserhalb der Expatriate-Lebenswelt geben könnten. Die Vereine könnten ihre Mitglieder nach einiger Zeit des Ankommens auf weitere Angebote vor Ort, die ausserhalb der International Community liegen, hinweisen und diese so systematisch vorbereiten. So könnten die international ausgerichteten Vereine selbst ihren Teil dazu beitragen, dass ihre Neumitglieder sich nach einer gewissen „Schon- oder Karenzzeit“ im geschützten Rahmen schliesslich anderen Engagement-Karrieren widmen, die ausserhalb der internationalen Vereine liegen. Hierbei ist aber darauf hinzuweisen, dass es durch solche Ansätze keinesfalls darum gehen soll, grundsätzlich alle Neumitglieder in Richtung zu einem lokalen Engagement hin zu bewegen bzw. zu beraten. Es soll vielmehr als eine Option für die Interessierten unter den Mitgliedern der internationalen Vereine angesehen werden, die dabei helfen kann, erste Hürden bei der Kontaktsuche zu lokalen Vereinen abzubauen. Dabei wäre es von Vorteil, wenn die Vereine die Erfahrungen ihrer „long time stayer“ miteinbeziehen, von denen sich viele (wie bspw. Herr Dieter aus meiner Studie) in lokalen Settings engagieren, sich aber auch nach vielen Jahren vor Ort noch immer in einem internationalen Umfeld bewegen (möchten). Gleiche Erfahrungswerte könnte man sich von den sogenannten ,mixed couples“ versprechen, von denen ebenfalls viele, auch nach langer Ortsansässigkeit, immer wieder im Umfeld der internationalen Vereine aktiv sind.

${ }^{51}$ Vgl. http://www.lets-talk-zug.ch 


\section{Öffnung der Vereine, Anpassung an neue Herausforderungen}

Der Kanton Zug verfügt über eine sehr grosse und vielfältige Vereinslandschaft, ein grosser Teil des öffentlichen Lebens findet dort in den lokalen Vereinen statt. Die von mir befragten Vereine sind sich alle dessen bewusst, dass sie durch ihre Aktivität, ihrem Engagement vor Ort, einen wichtigen Beitrag zum öffentlichen Leben und zum gesellschaftlichen Zusammenhalt leisten. Bezüglich der bisherigen Erfahrungen zum Engagement von Expatriates in den Vereinen liegen allerdings sehr heterogene Erfahrungen und Meinungen vor. Einige sind sich, wie gezeigt wurde, sehr bewusst, dass diese neue Zielgruppe neue oder zumindest andere Rahmenbedingung braucht, um in etablierten Schweizer Vereinen vor Ort aktiv zu werden. Andere wiederum sehen hier keinen Handlungsbedarf und verbinden damit vor allem die mühevolle Integration von fremdsprachigen Personen in gewachsene Vereinsstrukturen, welche sich vermutlich nicht lohnt, weil diese neuen Vereinsmitglieder ohnehin nicht lange bleiben würden.

Wenn es um Anpassungen der Vereine an neue Herausforderungen durch Expatriates geht, so sind damit in erster Linie die flexibleren Handhabungen von Vereinsstrukturen gemeint. Das bedeutet nicht, dass man als Verein nun zwangsweise alles auf zwei Sprachen durchführen und organisieren muss. Es ist eher gemeint, dass sich ein Verein, falls er sich diesen neuen Engagierten bewusster öffnen möchte, flexiblere Umgangsformen damit finden sollte, manche Vereinsabläufe auch auf Englisch erklären zu können oder ggf. manche Kurse spontan auf Englisch durchzuführen (je nach Vereinssetting, z. B. bei Sport- oder Musikvereinen sind es vermutlich auch gar nicht so viele komplexe Abläufe, die dort erläutert werden müssten). Vereine könnten beispielsweise Angebote wie Schnupper-Mitgliedschaften anbieten oder gezielt damit werben, zweisprachig zu sein bzw. englischsprachige Optionen anzubieten. Ähnlich wie ich schon bei den Expatriate-Vereinen vorgeschlagen habe, könnte auch hier auf die Erfahrungen einzelner Vereine in Zug zurückgegriffen werden, die ihre Vereinsabläufe bewusst zweisprachig organisiert haben. Denkbar wären z. B. Best-Practice-Beispiele aus dem Verein Epsilon, der zum Zeitpunkt der Gruppendiskussion kollektiv beschlossen hatte, ein sogenanntes Buddy-System (also ein Götti/Gotte für fremdsprachige Neumitglieder) im Verein zu installieren.

Eine weitere, eng mit Flexibilisierung von Umgangsformen in Vereinen verbundene Herausforderung ist die häufig anzutreffende zeitliche Befristung in den meisten Expatriate-Biografien. Damit verbunden ist eine grundsätzliche Ambivalenz von unterschiedlichen Interessen, die ich als eine der drei grundlegenden Herausforderungen bei dieser Thematik sehe. Es geht dabei um ein Investment in eine Mitgliedschaft im Bewusstsein des befristeten Expatriate-Life vor Ort. Hierbei kommt es darauf an, als Verein auf die zeitliche Begrenztheit des Aufenthalts möglichst flexibel reagieren zu können und den Expatriates trotz dieser unklaren Zeitvorgabe durchaus verantwortungsvolle Aufgaben - wie die bspw. von Frau Haller deutlich eingeforderten ,big things“ - zuzutrauen. Es wird also generell darum gehen, in diesem Bereich eher projektartige Organisationsformen des Engagements anzustreben ${ }^{52}$.

\footnotetext{
${ }^{52}$ Eine Entwicklung, die sich im Übrigen in den letzten Jahren generell im Bereich des Engagements beobachten lässt. (vgl. Samochowiec et al. 2018; Freitag et al. 2016; Kubisch/Störkle 2016; Cattacin 2006)
} 
Abschliessend möchte ich zu einer Haltung anregen, die bewusst mit dem vorhandenen Engagementpotenzial der Expatriates umgeht. Insbesondere vor dem Hintergrund von Mitgliederschwund und Nachwuchsproblemen in den Vereinen wäre das Potenzial von interessierten neu Zugewanderten zu erkennen und zu mobilisieren. Hierzu muss in den Vereinen allerdings ein Bewusstsein dafür geschaffen werden, dass Expatriates trotz ihres zeitlich limitierten Aufenthalts als Teil der sozialen Lebenswelt vor Ort wahrgenommen und als solche auch - wenn möglich - miteinbezogen werden.

Open Access Dieses Kapitel wird unter der Creative Commons Namensnennung 4.0 International Lizenz (http://creativecommons.org/licenses/by/4.0/deed.de) veröffentlicht, welche die Nutzung, Vervielfältigung, Bearbeitung, Verbreitung und Wiedergabe in jeglichem Medium und Format erlaubt, sofern Sie den/die ursprünglichen Autor(en) und die Quelle ordnungsgemäß nennen, einen Link zur Creative Commons Lizenz beifügen und angeben, ob Änderungen vorgenommen wurden.

Die in diesem Kapitel enthaltenen Bilder und sonstiges Drittmaterial unterliegen ebenfalls der genannten Creative Commons Lizenz, sofern sich aus der Abbildungslegende nichts anderes ergibt. Sofern das betreffende Material nicht unter der genannten Creative Commons Lizenz steht und die betreffende Handlung nicht nach gesetzlichen Vorschriften erlaubt ist, ist für die oben aufgeführten Weiterverwendungen des Materials die Einwilligung des jeweiligen Rechteinhabers einzuholen.

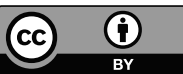

